\title{
The importance of immunization in immune-mediated inflammatory disease cannot be overstated
}

\author{
Sang Hyoung Park \\ Department of Gastroenterology and Inflammatory Bowel Disease Center, Asan Medical Center, University of Ulsan College of Medicine, Seoul, \\ Korea
}

\section{Article: Nonimmunity against hepatitis B virus infection in patients newly diagnosed with inflammatory bowel disease (Intest Res 2018;16:400-408)}

Inflammatory bowel disease (IBD) is an immune-mediated inflammatory disease (IMID) caused by the disruption of normal immune mechanisms. ${ }^{1}$ Patients with IBD are treated with anti-inflammatory drugs such as 5 -aminosalicylic acid, corticosteroids, immunomodulators (previously called immunosuppressants) such as thiopurine and methotrexate, and biologics including anti-tumor necrosis factor (antiTNF) agents, anti-integrin, and other small molecule drugs including anti-interleukin-12/23 or Janus kinase inhibitors. ${ }^{2}$ The long-term use of these drugs can lead to increased risk of adverse events such as opportunistic infections that are preventable with vaccination. ${ }^{3}$ Moreover, IBD itself is known to be associated with impaired innate mucosal immunity, although IBD patients are not routinely considered to be immunocompromised unless they are receiving concomitant therapy. ${ }^{3}$ Therefore, guidelines from gastroenterology societies recommend routine vaccination for preventable illnesses such as influenza, pneumonia, and hepatitis A and B infection in IBD patients, especially before starting immunosuppressive drugs. ${ }^{3,4}$

I read with great interest the article by Yeo et al. ${ }^{5}$ on nonimmunity against $\mathrm{HBV}$ infection in patients newly diagnosed with IBD in this issue of Intestinal Research. They enrolled

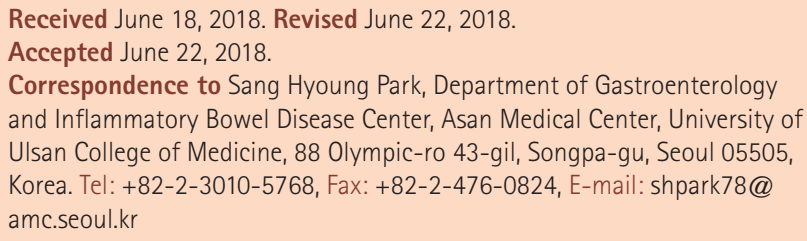

newly diagnosed IBD patients prospectively to check the immune status of HBV at the time of IBD diagnosis. In addition, they performed a case-control study with sex- and agematched controls to evaluate the risk factors for nonimmunity against HBV. The prevalence of chronic HBV infection and past infection were not different between the 2 groups (chronic HBV infection: IBD, $3.8 \%$ vs. controls, $4.9 \%, P=0.596$; past infection: IBD, $26.2 \%$ vs. controls, $28.8 \%, P=0.625)$. However, the rate of effective vaccination in IBD patients was significantly lower than that in controls (38.5\% in IBD vs. $48.0 \%$ in controls, $P=0.013$ ). This difference was more evident in patients with IBD who were diagnosed at less than age 20 (IBD, $41.5 \%$ vs. controls, $22.4 \% ; P=0.018$ ). In addition, in multivariate analysis, an age at diagnosis younger than 20 years $(P=0.024)$ and longer duration of symptoms before diagnosis $(P=0.027)$ were independent risk factors for nonimmunity to HBV. Based on these findings, the authors suggested screening for HBV serologic markers to establish appropriate vaccination plans in patients with IBD at diagnosis.

The novel finding of this study might be the high rate of nonimmunity to HBV in Korean IBD patients at the time of diagnosis, especially in those who are younger. The incidence and prevalence of IBD is still increasing in developing countries that are also in an HBV-endemic area such as Asia. In 2010, the estimated disease burden of IBD in India (1.4 million for IBD; 0.3 million for CD, and 1.1 million for UC) was almost similar to the disease burden of IBD in the United States (1.6 million for IBD; 0.8 million for CD, and 0.9 million for UC). ${ }^{6}$ In addition, immunomodulators and biologics have been used increasingly more frequently and

\footnotetext{
๑ Copyright 2018. Korean Association for the Study of Intestinal Diseases. All rights reserved.

This is an Open Access article distributed under the terms of the Creative Commons Attribution Non-Commercial License (http://creativecommons.org/licenses/by-nc/4.0)

which permits unrestricted non-commercial use, distribution, and reproduction in any medium, provided the original work is properly cited.
} 
earlier over the past decade in Asian patients with IBD. One study from Korea showed that the 5-year cumulative probability of prescription for thiopurines and anti-TNF agents was $28.9 \%$ and $1.4 \%$, respectively, in an older cohort (diagnosis of CD before 2000), but $88.1 \%$ and $23.7 \%$, respectively, in a recent cohort (diagnosis of CD after 2005) $(P<0.001){ }^{7}$ In addition, the usual age at diagnosis of IBD in Asian countries is the early 20s, especially for CD. A population-based study from Korea showed that the median age at diagnosis was 22 years for CD and 35 years for UC. ${ }^{8}$ Moreover, IBD patients with active HBV infection had a poor prognosis in a case-control study from Korea. ${ }^{9}$ Clinical outcomes such as hospitalization, surgery in UC, and mortality were worse in HBsAg-positive compared to HBsAg-negative IBD patients. ${ }^{9}$ For the above reasons, early screening and early detection of populations at risk for HBV infection should be emphasized in IMIDs including IBD.

There is a discrepancy regarding the timing of assessment for HBV status in IBD patients. Western guidelines such as the American College of Gastroenterology clinical guideline recommend testing for $\mathrm{HBV}$ infection (HBsAg, $\mathrm{HBcAb}$, and $\mathrm{HBsAb}$ ) and adequate vaccination of nonimmune patients before starting anti-TNF agents. ${ }^{4}$ However, the European Crohn's and Colitis Organisation consensus recommends that all IBD patients should be tested at diagnosis to assess HBV status. ${ }^{3}$ This difference between Western guidelines might be due to the low prevalence of $\mathrm{HBV}$ infection in these areas. In contrast, Asian guidelines recommend assessment of HBV serology at the time of IBD diagnosis. A consensus statement from China, which is an endemic area for HBV infection, said that all IBD patients should be tested for HBsAg, anti-HBs, and anti-HBc, and HBV screening is recommended when IBD is initially diagnosed. ${ }^{10}$ Taken together, screening for $\mathrm{HBV}$ infection should be considered in all IBD patients at the time of diagnosis, especially in Asian countries. In addition, adequate vaccination against HBV should be recommended to all newly diagnosed IBD patients. Thus, the importance of immunization in IBD cannot be overstated, given the findings of this study. To clarify this issue, a prospective study based on a multinational, large inception cohort is needed.

\section{FINANCIAL SUPPORT}

The authors received no financial support for the research, authorship, and/or publication of this article.

\section{CONFLICT OF INTEREST}

No potential conflict of interest relevant to this article was reported.

\section{AUTHOR CONTRIBUTION}

Writing and approval of final manuscript: SH Park.

\section{REFERENCES}

1. Baumgart DC, Sandborn WJ. Crohn's disease. Lancet 2012;380: 1590-1605.

2. Park SH, Aniwan S, Loftus EV Jr. Advances in the use of biologics and other novel drugs for managing inflammatory bowel disease. Curr Opin Pharmacol 2017;37:65-71.

3. Rahier JF, Magro F, Abreu C, et al. Second European evidencebased consensus on the prevention, diagnosis and management of opportunistic infections in inflammatory bowel disease. J Crohns Colitis 2014;8:443-468.

4. Farraye FA, Melmed GY, Lichtenstein GR, Kane SV. ACG clinical guideline: preventive care in inflammatory bowel disease. Am J Gastroenterol 2017;112:241-258.

5. Yeo SJ, Lee HS, Jang BI, et al. Nonimmunity against hepatitis B virus infection in patients newly diagnosed with inflammatory bowel disease. Intest Res 2018;16:400-408.

6. Singh P, Ananthakrishnan A, Ahuja V. Pivot to Asia: inflammatory bowel disease burden. Intest Res 2017;15:138-141.

7. Park SH, Yang SK, Park SK, et al. Long-term prognosis of Crohn's disease and its temporal change between 1981 and 2012: a hospital-based cohort study from Korea. Inflamm Bowel Dis 2014;20:488-494.

8. Yang SK, Yun S, Kim JH, et al. Epidemiology of inflammatory bowel disease in the Songpa-Kangdong district, Seoul, Korea, 1986-2005: a KASID study. Inflamm Bowel Dis 2008;14:542549.

9. Park SH, Yang SK, Lim YS, et al. Clinical courses of chronic hepatitis B virus infection and inflammatory bowel disease in patients with both diseases. Inflamm Bowel Dis 2012;18:20042010.

10. Inflammatory Bowel Disease Group; Chinese Society of Gastroenterology; Chinese Medical Association. Evidence-based consensus on opportunistic infections in inflammatory bowel disease (republication). Intest Res 2018;16:178-193. 